\title{
Performance of orthogonal space-time coded MCPM system in correlated channels
}

\author{
Wenli Shen, Yanping Yu \\ College of Information and Electronic Engineering, Zhejiang GongShang University, Hangzhou, China \\ Email: shenwenli@gmail.com, yuyanping@mail.zjgsu.edu.cn
}

\begin{abstract}
The paper investigates the average pair-wise error probability (PWEP) performance of orthogonal space-time coded MCPM (OST-MCPM) systems for two transmit antennas over independent and correlated channels. Simple upper boundary expression at high SNR is derived to evaluate the PWEP in correlated channel. The achievable diversity gain of this system decreases due to the signal correlation between the antennas. Simulation results show that the error performance over a correlated channel is degraded when the correlation coefficient increases. And the penalty on the code performance increases a lot in fully correlated channel. It can also be seen that the diversity gain decreases when the channel is fully correlated, which matches well with the theoretical analysis. The upper bounds can be looser when diversity order decreases. The effects of antenna spacing and azimuth power distribution parameters (angle of arrival/departure and angular spread) on the performance of OST-MCPM are also simulated.
\end{abstract}

\section{INTRODUCTION}

Space-time coding for multiple antennas has attracted much attention due to the potential capacity gain. It has been widely used as an efficient scheme to overcome the effects of multiple-path fading and increase the data rate for wireless communication systems [1]-[7]. Alamouti [2] proposed the design criterion of orthogonal space-time coding (OST) for two transmit antennas. This scheme achieves a full diversity gain with a simple maximum-likelihood decoding algorithm. Due to the orthogonality, the receiver can decode each transmitted signal separately. Tarokh et al. [5] extended this design rule to a general number of transmit antennas.

The multi-level continuous phase modulation (MCPM) has the characteristics of nonlinear phase, constant envelope, compact spectrum, high power efficiency and so on [8]-[10]. Therefore MCPM has become one of the main modulation schemes for the data transmission over both bandwidth- and power-limited channels such as mobile communications, satellite communications, data link and so on.

Space-time coded MCPM (STC-MCPM) is a technique that combines space-time coding with MCPM. It can not only provide power and bandwidth efficiency but also achieve diversity and coding gains without sacrificing data rate or bandwidth [11]. Since MCPM signals are continuous phase modulated waveforms but not regular coded data, Alamouti's design rule cannot be applied directly. Recently Wang et al. [12]-[14] extended Alamouti's orthogonal encoding criterion to MCPM signals and designed orthogonal space-time coded MCPM (OST-MCPM) systems.
In a multiple antenna system, insufficient antenna spacing, angle spread, and the lack of rich scattering may cause spatial correlation between antennas. A considerable work has been done to evaluate the impact of channel correlation on the performance of space-time code [15]-[21].

Most of the work published on STC-MCPM so far is on code design, and no similar work has been done on the influence of the spatially correlated MIMO channel. In this paper, the effect of the correlated channel on the diversity gain of this algorithm is investigated. The frame error rate (FER) performance of OST-MCPM systems in independent and identically distributed (i.i.d.) channels and spatially correlated channels are simulated and compared with each other. The effects of antenna spacing, angle of arrival/departure and angular spread on the performance of OST-MCPM are also investigated by simulation.

This paper is organized as follows: Section II first describes the system model of the spatially correlated OSTMCPM system. In Section III, the spatial correlation and the achievable diversity gain for the OST-MCPM system over Rayleigh fading channels are studied. Then simulation results and related discussions are presented in Section IV. Finally the conclusions are drawn in Section V.

Throughout this paper, we use the following notations. $A^{T}$ and $A^{H}$ stand for the transposition and the conjugate transpose of matrix $A$ respectively. $\operatorname{det}(A)$ is the determinant of $A, \operatorname{tr}(A)$ denotes the trace of matrix $A$. $I_{N}$ stands for the $N \times N$ identity matrix.

\section{System Model}

In this paper, a wireless communication system with two transmit antennas and $n_{R}$ receive antennas is considered. For simplification we just focus on the baseband equivalent block diagram of the correlated OST-MCPM system, which is shown in Fig. 1. The information bits $I$ are mapped to sequence $d \in\{ \pm 1, \pm 3, \cdots, \pm(M-1)\}$, as the input of space-time coded modulator. Then the orthogonal space-time coded MCPM signals are simultaneously transmitted from the two transmit antennas.

The transmitted signal at the $i$-th antenna can be represented as

$$
s_{i}(t, d)=\sqrt{\frac{2 E_{s}}{T}} \exp \left\{j \phi_{i}(t, d)\right\}, i=1,2 .
$$




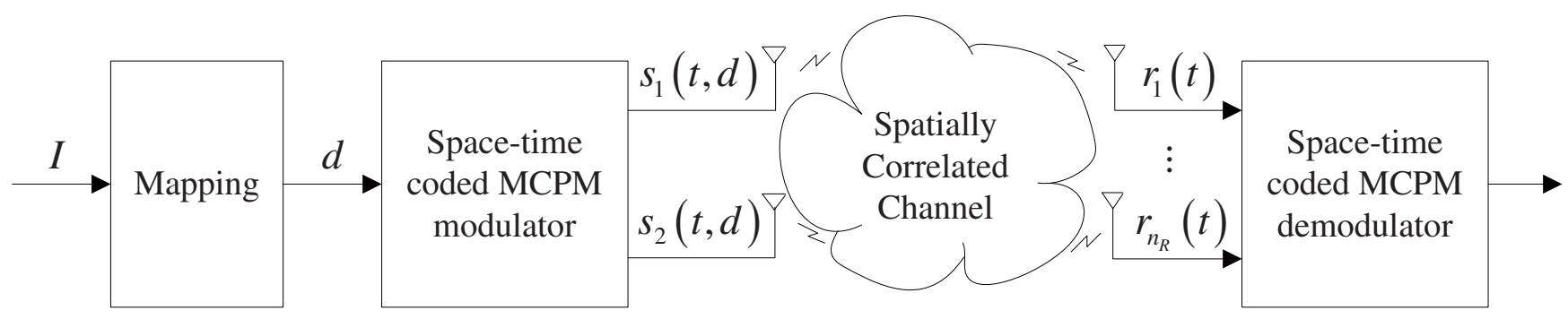

Fig. 1. Simplified base-band equivalent block diagram of correlated OST-MCPM system

$$
\phi_{i}(t, d)=2 \pi h \sum_{n=0}^{k} d_{i, n} q(t-n T), k T<t \leq(k+1) T .
$$

where $d=\left(d_{1,1}, d_{2,1}, \cdots, d_{1, k}, d_{2, k}\right), E_{s}$ is the symbol energy, $T$ is the symbol period, $\phi_{i}(t, d)$ is the carried phase information, $h$ is the modulation index. $q(t)=\int_{-\infty}^{t} g(\tau) d \tau$ is the phase smoothing response function. $g(t)$ is the pulse shaping function, which is nonzero only at the limited time period $0 \leq t \leq L T$ ( $L$ is the modulation memory), and it decides the smoothness of the transmitted phase.

Wang et al. extended Alamouti's encoding rule to CPM, where a design methodology was presented such that the signals $s_{1}(t, d)$ and $s_{2}(t, d)$ from the two transmit antennas over two symbol periods are orthogonal, i.e., the rows of the matrix

$$
\left[\begin{array}{ll}
s_{1}(t, d) & s_{1}(t+T, d) \\
s_{2}(t, d) & s_{2}(t+T, d)
\end{array}\right]
$$

are orthogonal for each $t$. In this paper, full response CPM signals $(L=1)$ are mainly considered. In order to ensure the orthogonality between two waveforms $s_{1}(t, d)$ and $s_{2}(t, d)$, a second phase smoothing response function $q_{0}(t)$ is introduced with $q(0)=q_{0}(0)=0, q(T)=q_{0}(T)=1 / 2$. The symbol $d_{1, k}$ and $d_{2, k}$ are jointly encoded.

At the time slot between $2(k-1) T$ and $2 k T$, the transmitted signals of the first antenna can be written as

$$
\begin{gathered}
s_{1}(t, d)=\sqrt{\frac{2 E_{s}}{T}} \exp \left\{j \phi_{1}(t, d)\right\} . \\
\phi_{1}(t, d)=\theta_{1}(2 k-2)+2 \pi h d_{1, k} q(t-(2 k-2) T), \\
\text { for }(2 k-2) T<t \leq(2 k-1) T ; \\
\phi_{1}(t, d)=\theta_{1}(2 k-1)+2 \pi h d_{2, k} q(t-(2 k-1) T), \\
\quad \text { for }(2 k-1) T<t \leq 2 k T . \\
\theta_{1}(2 k-2)=\phi_{1}((2 k-2) T, d), \\
\theta_{1}(2 k-1)=\phi_{1}((2 k-1) T, d) .
\end{gathered}
$$

At the time slot between $2(k-1) T$ and $2 k T$, the following signals are transmitted through the second antenna

$$
\begin{gathered}
s_{2}(t, d)=\sqrt{\frac{2 E_{s}}{T}} \exp \left\{j \phi_{2}(t, d)\right\} . \\
\phi_{2}(t, d)=\quad \theta_{2}(2 k-2)-2 \pi h d_{2, k} q(t-(2 k-2) T) \\
+2 \pi c_{k} q_{0}(t-(2 k-2) T), \\
\quad \text { for }(2 k-2) T<t \leq(2 k-1) T ; \\
\phi_{2}(t, d)=\quad \theta_{2}(2 k-1)-2 \pi h d_{1, k} q(t-(2 k-1) T) \\
+2 \pi c_{k} q_{0}(t-(2 k-1) T), \\
\text { for }(2 k-1) T<t \leq 2 k T .
\end{gathered}
$$

$$
\begin{gathered}
\theta_{2}(2 k-2)=\phi_{2}((2 k-2) T, d), \\
\theta_{2}(2 k-1)=\phi_{2}((2 k-1) T, d) . \\
c_{k}=1+2 h+2\left(\left(d_{1, k}+d_{2, k}\right) h / 2 \bmod 1\right)
\end{gathered}
$$

It can be checked that

$$
s_{1}(t, d) s_{2}^{*}(t, d)=-s_{1}(t+T, d) s_{2}^{*}(t+T, d)
$$

Consequently the row vectors of the transmitted signal matrix defined in (3) are orthogonal for each $t$.

\section{Performance analysis}

These algorithms are presented under the assumption that the channels are quasi-static flat Rayleigh fading, i.e. the fading coefficients are constant for the whole frame. The received signals at the $j$-th antenna can be written as

$$
r_{j}(t)=\sum_{i=1}^{2} h_{i, j} s_{i}(t, d)+n_{j}(t), j=1,2, \cdots, n_{R},
$$

where $h_{i, j}$ is the correlated fading coefficient between the $i$-th transmit antenna and the $j$-th receive antenna, and is modeled as a complex Gaussian random variable with variance $\sigma^{2}$. $n_{j}(t)$ is the additive white Gaussian noise (AWGN) at the $j$-th receive antenna, which is modeled as a zero-mean complex Gaussian random variable with power spectral density $N_{0}$ per dimension.

\section{A. Spatially Correlated Channel}

We assume that uniform linear array (ULA), which is an array that has all its elements on a line with equal spacing between the elements, is used for both the $n_{T}$ transmit and the $n_{R}$ receive antennas.

A slightly less general but more useful model considers correlations on transmit and receive sides separately. Representing the transmit-side correlation matrix as $R_{T}$ while receive-side correlation matrix as $R_{R}$. The inter-element spacings between the antennas at the transmit-side and receive-side are $d_{T}$ and $d_{R}$ respectively. The mean angle-of-departure (AOD) at the transmitter side and the mean angle-of-arrival (AOA) at the receiver side are denoted by $\theta_{T}$ and $\theta_{R}$. Then we define

$$
\rho\left(s d_{R}, \theta_{R}, \Delta_{R}\right)=E\left\{h_{i, j}, h_{i+s, j}^{*}\right\}, j=1,2, \cdots, n_{R} .
$$

to be the fading correlation coefficient between the two receive antenna placed $s d_{R}$ apart. $\Delta_{R}$ are the angular spreads. The transmit spatial correlation matrix $R_{T}$ could be defined 
similarly. Consequently the correlation matrix $R_{R}$ can be represented as

$$
\left[R_{R}\right]_{m, n}=\rho\left((n-m) d_{R}, \theta_{R}, \Delta_{R}\right) .
$$

The array propagation vectors can be represented as

$$
\begin{aligned}
& a_{T}\left(\theta_{T}^{l}\right)=\left[1, e^{-j 2 \pi d_{T} \sin \theta_{T}^{l} / \lambda}, \cdots, e^{-j 2 \pi\left(n_{T}-1\right) d_{T} \sin \theta_{T}^{l} / \lambda}\right]^{T}, \\
& a_{R}\left(\theta_{R}^{l}\right)=\left[1, e^{-j 2 \pi d_{R} \sin \theta_{R}^{l} / \lambda}, \cdots, e^{-j 2 \pi\left(n_{R}-1\right) d_{R} \sin \theta_{R}^{l} / \lambda}\right]^{T} .
\end{aligned}
$$

where $\theta_{T}^{l}$ and $\theta_{R}^{l}$ represent the actual AOD at the transmitter side and the AOA at the receiver side of the $l^{\text {th }}$ path. $\theta_{T}^{l} \in\left[\theta_{T}-\Delta_{T}, \theta_{T}+\Delta_{T}\right], \theta_{R}^{l} \in\left[\theta_{R}-\Delta_{R}, \theta_{R}+\Delta_{R}\right] . \lambda=c / f_{c}$ is the carrier frequency wavelength of a narrow-band signal with center frequency $f_{c}$.

Consequently the correlation matrix $R_{T}$ and $R_{R}$ could be represented as

$$
\begin{aligned}
& R_{T}=\frac{1}{L} \sum_{l=1}^{L} a_{T}\left(\theta_{T}^{l}\right) a_{T}^{H}\left(\theta_{T}^{l}\right) . \\
& R_{R}=\frac{1}{L} \sum_{l=1}^{L} a_{R}\left(\theta_{R}^{l}\right) a_{R}^{H}\left(\theta_{R}^{l}\right) .
\end{aligned}
$$

where $L$ denotes the number of the dominant unresolvable paths of the spatial scenario.

Finally, the correlated channel is represented as [22]

$$
H=R_{R}^{\frac{1}{2}} H_{w} R_{T}^{\frac{T}{2}}
$$

where $H_{w}$ is a i.i.d. channel fading coefficients matrix.

\section{B. The MLSD Algorithm}

The optimum decoding and demodulation of OST-MCPM system is a maximum likelihood sequence detection (MLSD) algorithm, which could be expressed as

$$
\hat{d}=\underset{d}{\arg \min }\left\{\sum_{j=1}^{n_{R}} \int_{0}^{N_{c} T}\left|r_{j}(t)-\sum_{i=1}^{2} h_{i, j} s_{i}(t, d)\right|^{2} d t\right\} .
$$

where $N_{c}$ is the symbol length of a frame.

The pair-wise error probability (PWEP) conditioned on $H$ can be approximated by [13]

$$
P\left(d_{1} \rightarrow d_{2} \mid H\right)=Q\left(\sqrt{\frac{E_{s}}{2 N_{0}} d^{2}\left[S\left(t, d_{1}\right), S\left(t, d_{2}\right)\right]}\right) .
$$

where $d^{2}\left[S\left(t, d_{1}\right), S\left(t, d_{2}\right)\right]$ is a modified Euclidean distance between the two space-time coded CPM signal matrices $S\left(t, d_{1}\right)$ and $S\left(t, d_{2}\right)$, given by

$$
\begin{aligned}
& d^{2}\left[S\left(t, d_{1}\right), S\left(t, d_{2}\right)\right] \\
& =\sum_{j=1}^{n_{R}} \int_{0}^{N_{c} T}\left|\sum_{i=1}^{2} h_{i, j}\left[s_{i}\left(t, d_{1}\right)-s_{i}\left(t, d_{2}\right)\right]\right|^{2} d t .
\end{aligned}
$$

Let

$$
\Gamma=d^{2}\left[S\left(t, d_{1}\right), S\left(t, d_{2}\right)\right]
$$

By using Graig's formula for the Gaussian $Q$ function

$$
Q(x)=\frac{1}{\pi} \int_{0}^{\frac{\pi}{2}} \exp \left\{-\frac{x^{2}}{2 \sin ^{2} \theta}\right\} d \theta .
$$

we can rewrite the conditional PWEP

$$
P\left(d_{1} \rightarrow d_{2} \mid H\right)=\frac{1}{\pi} \int_{0}^{\frac{\pi}{2}} \exp \left\{-\frac{E_{s}}{4 N_{0} \sin ^{2} \theta} \Gamma\right\} d \theta .
$$

In order to calculate the average PWEP, we average (24) with respect to the distribution of $\Gamma$. The average PWEP can be represented in terms of the moment generating function (MGF) of $\Gamma$, which is given by

$$
M_{\Gamma}(s)=\int_{0}^{\infty} e^{s \Gamma} P_{\Gamma}(\Gamma) d \Gamma .
$$

Thus the average PWEP can be represented as

$$
\begin{aligned}
& P\left(d_{1} \rightarrow d_{2}\right)=\frac{1}{\pi} \int_{0}^{\frac{\pi}{2}} E\left[\exp \left(-\frac{E_{s}}{4 N_{0} \sin ^{2} \theta} \Gamma\right)\right] d \theta \\
& =\frac{1}{\pi} \int_{0}^{\frac{\pi}{2}} \int_{0}^{\infty} \exp \left(-\frac{E_{s}}{4 N_{0} \sin ^{2} \theta} \Gamma\right) P_{\Gamma}(\Gamma) d \Gamma d \theta \\
& =\frac{1}{\pi} \int_{0}^{\frac{\pi}{2}} M_{\Gamma}\left(-\frac{E_{s}}{4 N_{0} \sin ^{2} \theta}\right) d \theta .
\end{aligned}
$$

Equation (21) can be rewritten as

$$
d^{2}\left[S\left(t, d_{1}\right), S\left(t, d_{2}\right)\right]=\operatorname{tr}\left(H B\left(d_{1}, d_{2}\right) H^{H}\right) .
$$

where $B\left(d_{1}, d_{2}\right)$ is a $2 \times 2$ difference matrix with entries

$$
\begin{aligned}
{\left[B\left(d_{1}, d_{2}\right)\right]_{m, n} } & =\int_{0}^{N_{c} T}\left[s_{m}\left(t, d_{1}\right)-s_{m}\left(t, d_{2}\right)\right] \\
& \times\left[s_{n}\left(t, d_{1}\right)-s_{n}\left(t, d_{2}\right)\right]^{*} d t .
\end{aligned}
$$

Therefore the PWEP can be represented as [20]

$$
\begin{aligned}
P\left(d_{1} \rightarrow d_{2}\right) & =\frac{1}{\pi} \int_{0}^{\frac{\pi}{2}} \prod_{i=1}^{2} \prod_{j=1}^{n_{R}}\left(1+\frac{E_{s}}{4 N_{0} \sin ^{2} \theta} \mu_{i} \lambda_{j}\right)^{-1} d \theta \\
& \leq \prod_{i=1}^{2} \prod_{j=1}^{n_{R}}\left(1+\frac{E_{s}}{4 N_{0}} \mu_{i} \lambda_{j}\right)^{-1} .
\end{aligned}
$$

where $\mu_{i}$ and $\lambda_{j}$ are the eigenvalues of $B\left(d_{1}, d_{2}\right) R_{T}$ and $R_{R}$. Equation (29) is the Chernoff bound for the PWEP. When the SNR is high, the upper bound can be simplified as

$$
P\left(d_{1} \rightarrow d_{2}\right) \leq\left(\frac{E_{s}}{4 N_{0}}\right)^{-r \hat{r}} \prod_{i=1}^{r} \prod_{j=1}^{\hat{r}}\left(\mu_{i} \lambda_{j}\right)^{-1} .
$$

where $r, \hat{r}$ are the ranks of $B\left(d_{1}, d_{2}\right) R_{T}$ and $R_{R}$ respectively. $\mu_{i}(i=1,2, \cdots, r)$ and $\lambda_{j}(j=1,2, \cdots, \hat{r})$ are the nonzero eigenvalues of $B\left(d_{1}, d_{2}\right) R_{T}$ and $R_{R}$. In the high-SNR regime, the diversity order of a pair of codewords of the OST-MCPM system is the exponent of SNR, i.e., $r \hat{r}=\operatorname{rank}\left(B\left(d_{1}, d_{2}\right) R_{T}\right)$. $\operatorname{rank}\left(R_{R}\right)$.

For the special case of fully correlated channel where $\left(R_{T}\right)_{i, j}=\left(R_{R}\right)_{i, j}=1$, the diversity order $r \hat{r}$ is 1 . For i.i.d. channels, the spatially correlated channels are $R_{T}=I_{n_{T}}$, 
$R_{R}=I_{n_{R}}$. Consequently, the achievable diversity gain could be represented as $r \hat{r}=\operatorname{rank}\left(B\left(d_{1}, d_{2}\right) R_{T}\right) \cdot n_{R}$.

The typical scenario in the wireless channel environment is partial channel correlation. The channel correlation matrices $R_{T}$ and $R_{R}$ are nonnegative definite Hermitian matrices of a Toeplitz form. $R_{T}=R_{T}^{H}, \operatorname{det}\left(R_{T}\right)>0$ and $R_{R}=R_{R}^{H}, \operatorname{det}\left(R_{R}\right)>$ 0 . Obviously the correlation matrices $R_{T}$ and $R_{R}$ will always have full rank. Thus the diversity order is the same as that in i.i.d. channels.

In order to achieve full diversity, $B\left(d_{1}, d_{2}\right)$ should be full rank. The waveform difference from the two transmit antennas should be linearly independent over the complex field. That is to say, for any complex numbers $C_{1}$ and $C_{2}$, there exists a $t$, such that [13]

$$
C_{1}\left[s_{1}\left(t, d_{1}\right)-s_{1}\left(t, d_{2}\right)\right]+C_{2}\left[s_{2}\left(t, d_{1}\right)-s_{2}\left(t, d_{2}\right)\right] \neq 0 .
$$

unless $C_{1}=C_{2}=0$.

Because the transmitted signals from the two antennas are orthogonal, the transmitted signal matrix $S(t, d)$ is unitary. Thus it is easy to see that the OST-MCPM satisfies the above condition of full rank. Therefore, in i.i.d. or partially correlated channels the OST-MCPM system can achieve full diversity.

\section{Simulation Results}

In this section, some simulation results are presented to evaluate the FER performances of the MLSD over quasi-static Rayleigh fading channels with spatial correlation. The frame error rate (FER) results of this algorithm are plotted against $E_{b} / N_{0}$. OST-MCPM systems of full response CPM signals with two transmit antennas are considered. The number of receive antennas is one and two. The modulation level $M$ is chosen to be 2 and 4 , while the modulation index $h=1 / M$. The pulse shaping functions $g(t)$ and $g_{0}(t)$ are chosen to be 1 REC and 1RC [9] respectively in both OST-2CPM and OST4CPM systems. The transmit and receive antenna correlation matrices are given by

$$
R_{T}=\left[\begin{array}{cc}
1 & \rho_{t} \\
\rho_{t} & 1
\end{array}\right], R_{R}=\left[\begin{array}{cc}
1 & \rho_{r} \\
\rho_{r} & 1
\end{array}\right],
$$

for the two transmit and two receive antennas systems. The antenna spacing and azimuth power distribution parameters at the receiver side are chosen as

$$
d_{R}=0 \sim 3 \lambda, \theta_{R}=22.5^{\circ}, \Delta_{R}=\left[5^{\circ}, 35^{\circ}, 180^{\circ}\right] .
$$

\section{A. Effect of Correlation}

Fig. 2, Fig. 3 and Fig. 4 show the FER performance of the MLSD algorithm for OST-2CPM and OST-4CPM systems over correlated fading channels. It can be seen from these figures that the system in i.i.d. MIMO channels $\left(\rho_{t}=\rho_{r}=0\right)$ has the same diversity gain as that of the system over partially correlated channels $\left(0<\rho_{t}<1,0<\rho_{r}<1\right)$. Simulation results show that the error performance over a correlated channel is degraded by approximately $2 \mathrm{~dB}$ at a FER of $10^{-2}$ when the correlated index $\rho_{t}$ increases from 0 to 0.7 for the one-receive OST-2CPM system. And the penalty on the code performance increases to over $9 \mathrm{~dB}$ at the same FER in fully correlated

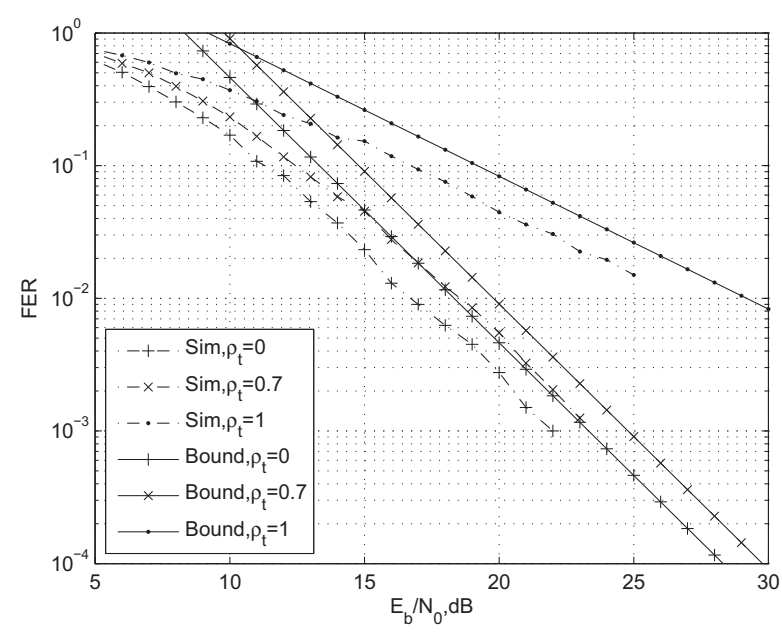

Fig. 2. FER curves of MLSD of OST-2CPM systems $\left(n_{R}=1\right)$.

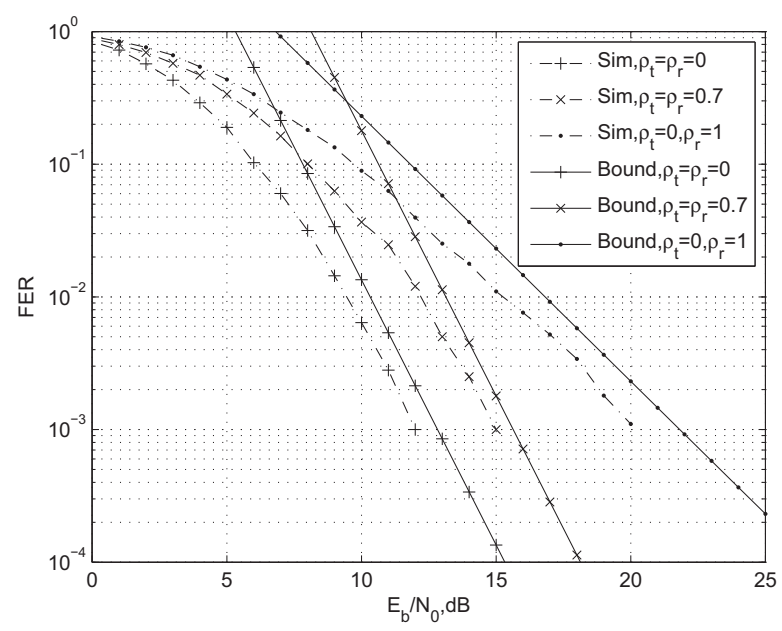

Fig. 3. FER curves of MLSD of OST-2CPM systems $\left(n_{R}=2\right)$.

channel. From Fig. 3 we can see that the performance is degraded by approximately $2.7 \mathrm{~dB}$, when the correlated indexes $\rho_{t}$ and $\rho_{r}$ increase to 0.7 .

It can also be observed from the three figures that the diversity gain decreases when the channel is fully correlated, which matches well with the theoretical analysis. The upper bounds can be looser whenever diversity is low, e.g., if the number of receive antennas is small, or the transmit or receive antennas are fully correlated.

\section{B. Effect of Antenna Spacing}

First we consider the effect of receive antenna spacing on the FER performance of the MLSD algorithm for OST-2CPM and OST-4CPM systems. Consider a system with two receive antennas. For simplicity, it is assumed that the scattering environment at the transmitter side is uncorrelated due to the sufficiently long distance between the transmit antennas. 


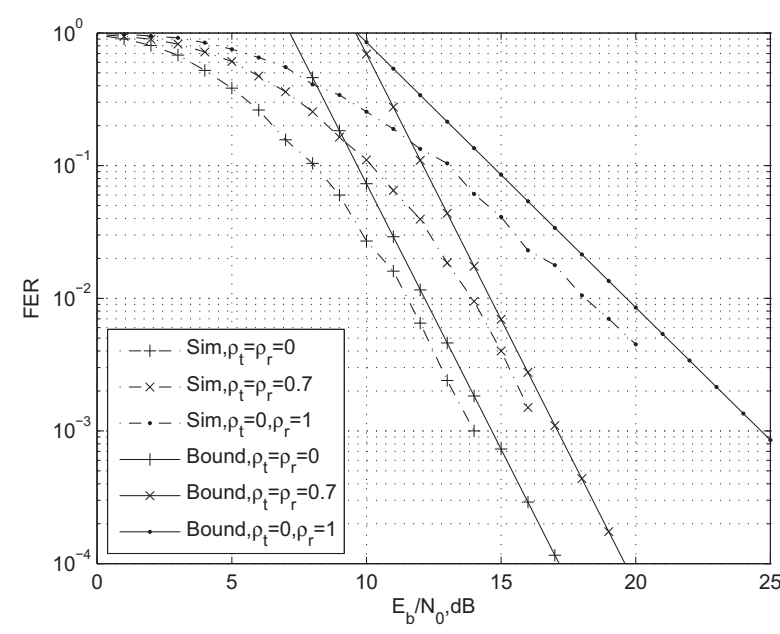

Fig. 4. FER curves of MLSD of OST-4CPM systems $\left(n_{R}=2\right)$.

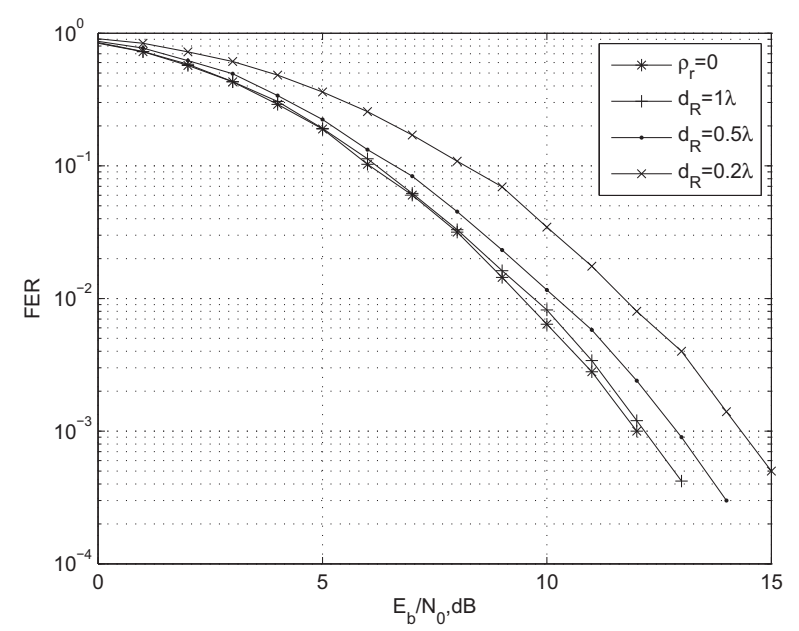

Fig. 5. FER curves of MLSD of OST-2CPM systems $\left(n_{R}=2, \theta_{R}=\right.$ $22.5^{\circ}, \Delta_{R}=35^{\circ}$ ).

Fig. 5 and Fig. 6 show the FER performance with different receive antennas separation $\left(d_{R}=[1 \lambda, 0.5 \lambda, 0.2 \lambda]\right)$. The mean AOA $\theta_{R}$ at the receiver side is chosen to be $22.5^{\circ}$. The angular spread $\Delta_{R}$ is $35^{\circ}$.

It can be observed from the figures that the FER performance degradation is not obvious, compared with the FER for the i.i.d slow fading channel when the receive antenna separation is $0.5 \lambda$ or higher. However the effect of antenna separation is significant when the receive antenna separation is small.

\section{Effect of Angular Spread}

Fig. 7 and Fig. 8 illustrate the effects of receive angular spreads $\left(\Delta_{R}=\left[0^{\circ}, 35^{\circ}, 180^{\circ}\right]\right)$ on the FER performance of the MLSD algorithm for OST-2CPM and OST-4CPM systems with mean AOA $\theta_{R}=22.5^{\circ}$.

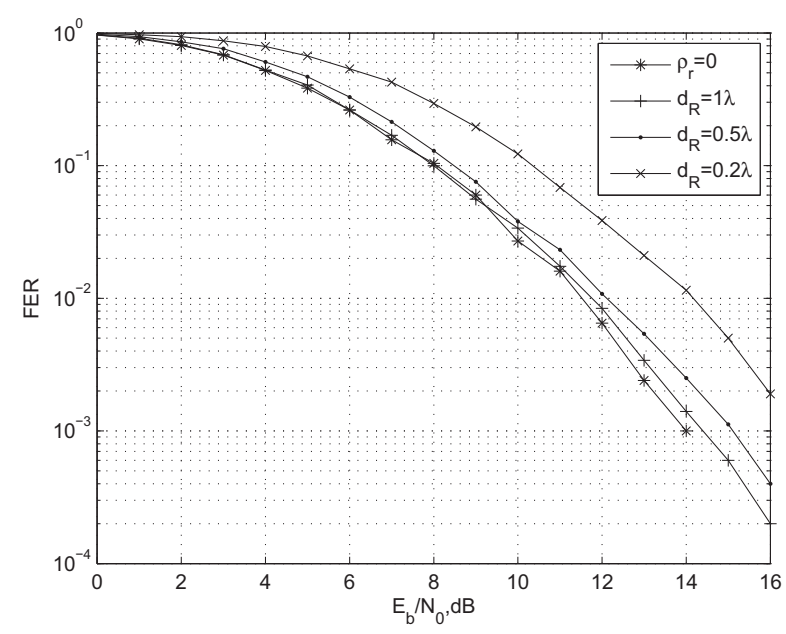

Fig. 6. FER curves of MLSD of OST-4CPM systems $\left(n_{R}=2, \theta_{R}=\right.$ $22.5^{\circ}, \Delta_{R}=35^{\circ}$ ).

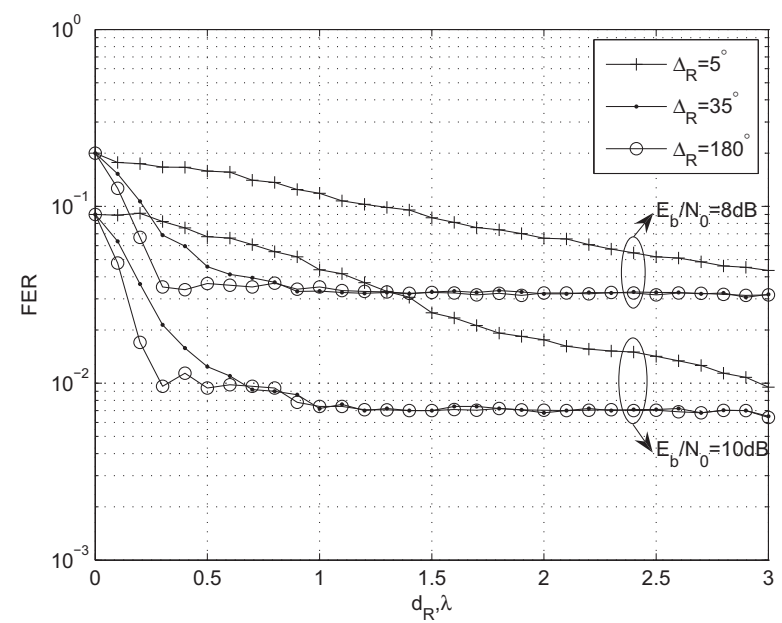

Fig. 7. FER curves of MLSD of OST-2CPM systems $\left(n_{R}=2, \theta_{R}=22.5^{\circ}\right)$.

From the two figures, we can observe that for a given $E_{b} / N_{0}$, the FER performances are improved with the increase of the receive angular spread and the receive antenna separation. However, the performances don't improve monotonically as the receive antenna separation are increased. They do not change a lot with medium or high angular spreads when the receive antennas are spaced more than $0.5 \lambda$ apart. When the angular spread is quite small (e.g. $\Delta_{R}=5^{\circ}$ ), the two receive antennas need to be placed at least several wavelengths apart to achieve acceptable performances.

\section{Conclusion}

The effect of the spatial correlation on MLSD of OSTMCPM system over Rayleigh fading channels was investigated in the paper. The PWEP upper bound of OST-MCPM systems was deduced to evaluate the achievable diversity gain over quasi-static Rayleigh fading channels with spatial correlation. 


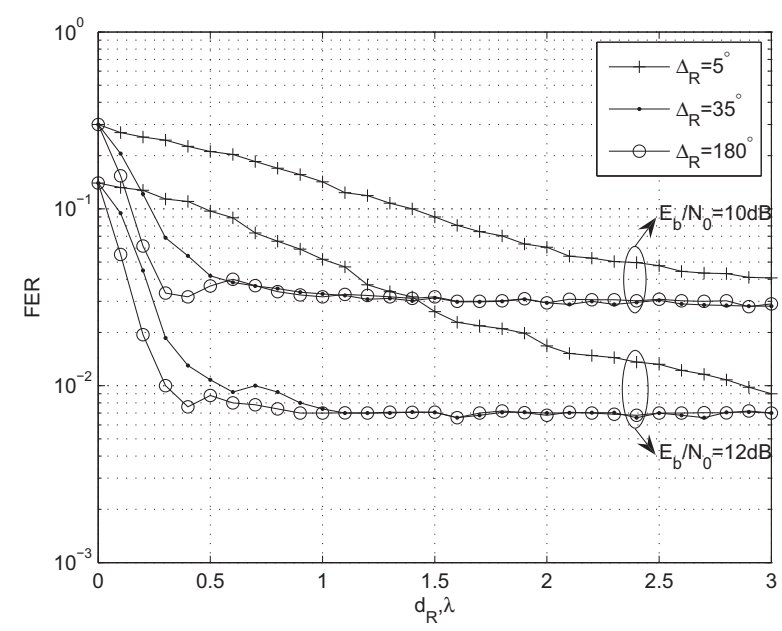

Fig. 8. FER curves of MLSD of OST-4CPM systems $\left(n_{R}=2, \theta_{R}=22.5^{\circ}\right)$.

Due to the orthogonality and the constant envelope, the OSTMCPM system in partially correlated channels still could achieve full diversity. The simulation results well match the theoretical analysis. The effects of antenna spacing and azimuth power distribution parameters (angle of arrival/departure and angular spread) on the FER performance of OST-MCPM are also simulated to estimate the error performance under given parameters.

\section{ACKNOWLEDGMENT}

The authors would like to thank the work members for their valuable comments that have improved the clarity of this paper. And this work is supported by Zhejiang Provincial Natural Science Foundation of China under Grant No.Y1090232.

\section{REFERENCES}

[1] G. Foschini, "Layered space-time architecture for wireless communication in a fading environment when using multiple antennas," Bell Labs Technical Journal, vol. 1, no. 2, pp. 41-59, 1996.

[2] S. Alamouti, "A simple transmit diversity technique for wireless communications," Selected Areas in Communications, IEEE Journal on, vol. 16 , no. 8, pp. 1451-1458, 1998.

[3] V. Tarokh, N. Seshadri, and A. Calderbank, "Space-time codes for high data rate wireless communication: performance criterion and code construction," Information Theory, IEEE Transactions on, vol. 44, no. 2 , pp. 744-765, 1998.

[4] V. Tarokh, A. Naguib, N. Seshadri, and A. Calderbank, "Space-time codes for high data rate wireless communication: performance criteria in the presence of channel estimation errors, mobility, and multiple paths," Communications, IEEE Transactions on, vol. 47, no. 2, pp. 199-207, 1999.

[5] V. Tarokh, H. Jafarkhani, and A. Calderbank, "Space-time block codes from orthogonal designs," Information Theory, IEEE Transactions on, vol. 45, no. 5, pp. 1456-1467, 1999.

[6] A. Naguib, V. Tarokh, N. Seshadri, and A. Calderbank, "A space-time coding modem for high-data-rate wirelesscommunications," Selected Areas in Communications, IEEE Journal on, vol. 16, no. 8, pp. 14591478, 1998.

[7] S. Baro, G. Bauch, and A. Hansmann, "Improved codes for space-time trellis-coded modulation," Communications Letters, IEEE, vol. 4, no. 1, pp. 20-22, 2000.

[8] T. Aulin, N. Rydbeck, and C. Sundberg, "Continuous Phase ModulationPart I and Part II,” IEEE Trans. Commun, vol. 29, no. 8, pp. 196-225, 1981.
[9] J. Proakis, Digital Communications. McGraw-Hill, 1983.

[10] J. Anderson, C. Sundberg, and T. Aulin, Digital Phase Modulation. Springer, 1986

[11] X. Zhang and M. Fitz, "Space-time code design with continuous phase modulation," Selected Areas in Communications, IEEE Journal on, vol. 21, no. 5, pp. 783-792, 2003.

[12] G. Wang and X. Xia, "An orthogonal space-time coded CPM system with fast decoding for two transmit antennas," Information Theory, IEEE Transactions on, vol. 50, no. 3, pp. 486-493, 2004.

[13] D. Wang, G. Wang, and X. Xia, "An orthogonal space-time coded partial response CPM system with fast decoding for two transmit antennas," Wireless Communications, IEEE Transactions on, vol. 4, no. 5, pp. 2410-2422, 2005.

[14] G. Wang, W. Su, and X. Xia, "Orthogonal-like space-time coded CPM system with fast decoding for three and four transmit antennas," Proc. Global Telecommunications Conf.(GLOBECOM), pp. 3321-3325.

[15] H. Bolcskei and A. Paulraj, "Performance of space-time codes in the presence of spatial fading correlation," Signals, Systems and Computers, 2000. Conference Record of the Thirty-Fourth Asilomar Conference on, vol. 1,2000

[16] M. Uysal and C. Georghiades, "Effect of spatial fading correlation on performance of space-time codes," Electronics Letters, vol. 37, no. 3, pp. 181-183, 2001

[17] M. Damen, A. Abdi, and M. Kaveh, "On the effect of correlated fading on several space-time coding and detection schemes," Vehicular Technology Conference, 2001. VTC 2001 Fall. IEEE VTS 54th, vol. 1, 2001.

[18] A. Dogandzic, "Chernoff bounds on pairwise error probabilities of space-time codes," Information Theory, IEEE Transactions on, vol. 49, no. 5, pp. 1327-1336, 2003.

[19] M. Uysal and C. Georghiades, "On the error performance analysis of space-time trellis codes," Wireless Communications, IEEE Transactions on, vol. 3, no. 4, pp. 1118-1123, 2004.

[20] A. Hedayat, H. Shah, and A. Nosratinia, "Analysis of space-time coding in correlated fading channels," Wireless Communications, IEEE Transactions on, vol. 4, no. 6, pp. 2882-2891, 2005.

[21] B. Clerckx and C. Oestges, "Finite-SNR Performance Analysis of Space-Time Coding in Correlated Ricean MIMO Channels," Information Theory, IEEE Transactions on, vol. 53, no. 12, pp. 4761-4777, 2007.

[22] C. Chuah, D. Tse, J. Kahn, and R. Valenzuela, "Capacity scaling in MIMO wireless systems under correlated fading," Information Theory, IEEE Transactions on, vol. 48, no. 3, pp. 637-650, 2002. 